\title{
Doppio framing, indugi e soglie narrative
}

Tre autori a confronto: Celati, Ghirri e Antonioni

\section{Cecilia Monina}

\section{OpenEdition}

\section{Journals}

Edizione digitale

URL: https://journals.openedition.org/cher/725

DOI: $10.4000 /$ cher.725

ISSN: 2803-5992

\section{Editore}

Presses universitaires de Strasbourg

\section{Edizione cartacea}

Data di pubblicazione: 9 juillet 2020

Paginazione: 123-134

ISBN: 979-10-344-0068-3

ISSN: 1968-035X

\section{Notizia bibliografica digitale}

Cecilia Monina, «Doppio framing, indugi e soglie narrative», reCHERches [Online], 24 | 2020, online dal 20 septembre 2021, consultato il 18 novembre 2021. URL: http://journals.openedition.org/cher/725 ; DOI: https://doi.org/10.4000/cher.725 


\title{
Doppio framing, indugi e soglie narrative Tre autori a confronto: Celati, Ghirri e Antonioni
}

\author{
CeCILIA Monina ${ }^{1}$
}

$\mathrm{I}^{1}$ concetto di doppio framing, o doppia cornice, è strettamente legato a quello di campo, sia esso visivo, cinematografico o narrativo. Si tratta di una cornice, non necessariamente fisica, che coinvolge autore e fruitore dell'opera e forza a una presa di posizione: si può guardare all'interno o rimanere fuori, esitare nella riflessione o mantenere, al contrario, un distacco.

La dicotomia alla base di quest'idea è la dialettica «dedans-dehors» proposta dal filosofo Gaston Bachelard nel suo La poetica dello spazio (Bachelard 1975: 247). I termini dentro e fuori richiamano la nozione spaziale almeno quanto il «dramma della geometria intima» (Bachelard 1975: 253): due spazi in rapporto tra loro e delimitati da una soglia che è la barriera, solo apparente, con cui l'individuo si scontra e davanti alla quale dovrà arrivare a una risoluzione.

I lavori di Antonioni, Celati e Ghirri sono stati spesso accostati e letti in prospettiva comparatistica per riflettere, in primo luogo, sul legame tra narrazione e paesaggio e sulla possibilità di raccontare un luogo e il suo immaginario, come i tre hanno saputo fare con l'Emilia e il Delta del Po. Se l'insistenza celatiana sull'elemento visivo, poi, nasce «a contatto con l'esperienza fotografica di Ghirri» (Bazzocchi 2012: 123) e trova la sua massima espressione nei documentari degli anni Novanta, i punti di contatto con l'opera di Michelangelo Antonioni possono apparire invece meno intuitivi, se si esclude il tema del territorio indagato con Gente del Po, breve documentario del 1943 nonché primo lavoro del regista ferrarese. Il doppio framing è un mezzo di cui i tre gli autori si sono serviti - seppur in tempi e contesti differenti - con esiti simili, e in queste pagine mi propongo di mostrare in che modo l'uso della doppia cornice sia stato declinato dai tre, con quali possibili intenzioni e quali risultati.

Le foto di Luigi Ghirri sono un buon esempio per introdurre e chiarire questo concetto. Pensiamo ad alcuni celebri scatti, diversi dei quali raccolti in

1 Cecilia Monina, Sorbonne Université. 
Kodachrome e altri libri, circa un decennio più tardi, prima in Paesaggio italiano e poi ne Il profilo delle nuvole. La grande cornice di legno bianco che serviva da supporto per tende da spiaggia (Marina di Ravenna, 1986 in Paesaggio italiano), la palizzata che, dal patio della casa, apre sul fitto di una boscaglia (Boretto, 1986 ne Il profilo delle nuvole), oppure l'affaccio metallico sul mare dell'île Rousse (Île Rousse, 1976 in Kodachrome). Tutte le immagini fin qui citate sono un esempio di doppio framing: oltre alla consueta cornice rettangolare entro cui siamo soliti pensare e ricevere una fotografia, appare in questi casi evidente la presenza di una seconda cornice, questa volta interna allo scatto, che necessariamente dirige e orienta lo sguardo. Il nostro occhio di spettatori segue, d'istinto, questa inquadratura interna alla foto quasi fosse lì a celare qualcosa, come se oltre quello spazio ricavato nell'immagine vi fosse un segno per forza degno di essere colto, osservato e compreso. La cornice, nel tempo, è stata pensata nel suo ruolo di margine e delimitazione, quindi di dispositivo simile alla finestra, in grado di canalizzare lo sguardo del fruitore nella direzione dell'opera e per questo funzionale alla rappresentazione - Simmel parla di «traviamento» (Simmel 1997: 213) - e come spazio stesso della rappresentazione, capace «di presentarsi come spazio della finzione, della mimesis, della figurazione di un altrove» (Somaini 2000).

E proprio qui risiede anche il senso della doppia cornice: è un'apertura, sì, ma verso qualcosa che siamo solamente liberi di immaginare o interpretare senza limitazioni e che dà avvio a una meta-rappresentazione. Così avviene che l'osservazione di una fotografia diventi anche un momento di indugio, di riflessione. La doppia cornice costringe a una sosta, obbliga cioè lo spettatore a fermarsi, a guardare all'interno per darsi la possibilità di concepire una realtà ulteriore.

Nella sua lezione di fotografia del 20 ottobre 1989, dedicata alla trasparenza, Ghirri scrive:

La fotografia non si ferma, non si esaurisce nell'oggetto di partenza, nel soggetto ripreso. Il risultato finale, l'esito che contempla chi fa la fotografia e chi successivamente la osserva è un ulteriore tipo di realtà, ottenuta attraverso strumenti, appunto, trasparenti. (Ghirri 2010: 145)

Fotografo e spettatore entrano in comunicazione, dando avvio a un dialogo tacito e di identificazione. L'uso della cornice può essere, in questo senso, uno strumento utile a rafforzare la relazione tra emittente e ricevente dell'opera, persino invertendone i ruoli:

nei dipinti con cornici di porte, di finestre o di nicchie chi guarda è chiamato a vedere l'immagine con gli occhi dell'artista/emittente. Egli si trova davanti alla situazione di emissione. Nei quadri con cornice finta, è il pittore a sdoppiarsi, mettendo se stesso (e la propria opera) nella situazione di ricezione. In entrambi i casi, i limiti dell'immagine vengono forzati. I rispettivi ruoli dell'artista e di chi guarda si suppone siano, in un modo o nell'altro, intercambiabili. (Stoichita 1998: 65) 
Quanto Stoichita sottolinea per il pittore, vale anche per il fotografo: egli deve quindi abbandonare i codici, evitare di porsi come semplice riproduttore e "guardare alla fotografia come a un modo di relazionarsi col mondo» (Ghirri 2010: 21). Quando scatta, il fotografo si trova sulla soglia: è lui a decidere cosa includere nella fotografia e cosa, invece, relegare all'esterno. Arbitrariamente, quindi, ritaglia una porzione di realtà, prendendola dalla disponibilità del campo visivo, e lascia fuori qualcosa, escludendolo di conseguenza:

Insieme al controllo dei rapporti cromatici, alle scelte di luce e alla profondità di campo, è molto importante riuscire a trovare il punto di equilibrio tra quello che includeremo nell'immagine e quello che rimarrà fuori. La fotografia è sempre un escludere il resto del mondo per farne vedere un pezzettino. (Ghirri 2010: 53)

Con l'uso della doppia cornice, lo spettatore può a sua volta porsi sulla soglia, provare l'esperienza dell'esercizio del fotografo, immedesimarsi e concentrarsi sull'inquadratura come apertura del proprio sguardo sulla realtà. Ghirri era solito utilizzare delle inquadrature naturali, «spazi nei quali il nostro sguardo è già guidato e orientato» (Ghirri 2010: 157), ricercandole nel paesaggio. O meglio, sfruttando quelle che trovava nelle campagne, ma anche negli elementi architettonici delle città: le colonne dei cancelli, gli archi, le saracinesche, le strutture vuote, le linee spigolose dei monumenti o degli edifici. Ammetteva in modo limpido, quasi a smascherare i trucchi del suo lavoro, di utilizzare escamotage come l'inserimento di quinte o di cercare nella realtà «quadri che appaiono come scenografie già costruite» (Ghirri 2010: 54) così che il processo di scarto e inclusione fosse manifesto. Questi elementi, paragonabili al mirino di una macchina, sono capaci di indirizzare e riportare un ordine dello sguardo.

Nella giornata del 19 gennaio 1990, Ghirri fa una premessa teorica al concetto di soglia, a cui parte della lezione sarà consacrata ${ }^{2}$, e decide di superare le definizioni teoriche fino a quel momento proposte. Ghirri aveva fatto della soglia un elemento di ricerca, pensando a questa nozione come a un principio del linguaggio fotografico. Un fondamento, quindi, che non è possibile ridurre o appiattire all'idea di una linea di demarcazione tra un ambiente chiuso e l'esterno, ma da intendersi invece in senso metaforico, come allegoria del confine e punto di equilibrio tra il nostro pensiero - la nostra rappresentazione delle cose, nonché conseguenza di una visione soggettiva del reale - e l'osservazione comune.

Nel momento in cui io scatto, mi trovo sulla soglia, sono sul punto di avvertire la possibilità di filtrare il mio interno con l'esterno. Devo fare una valutazione esatta, comunque un calcolo che so essere molto importante, che riguarda quello che deve essere tralasciato e quello che deve essere compreso. Tenere presente questo, quest'idea della soglia della rappresentazione reale, della soglia dell'inquadratura [...]. (Ghirri 2010: 154-155)

2 Ghirri fa riferimento alla definizione avanzata da Christian Norberg-Schulz nel volume del 1979 Genius Loci. Paesaggio ambiente architettura, testo in cui la soglia è definita dallo studioso come elemento «che identifica il punto di passaggio tra il mondo interno e il mondo esterno» (Norberg Schulz 1979: 12). 
La difficoltà maggiore consiste invece nel «superare la riproduzione della soglia e farla diventare un elemento sia dello spazio che del tempo» (Ghirri 2010: 161), ricercando quindi non l'inquadratura perfetta, ma quella già delineata, quell'inquadratura naturale citata in precedenza. Scrive Ghirri: «La macchina fotografica, semplicemente, dovrebbe essere una sottolineatura di queste indicazioni già presenti, di questi punti di attraversamento tra un interno e un esterno» (Ghirri 2010: 162-163). Si deve creare una relazione organica tra il soggetto e lo spazio, deve venire a delinearsi ciò di cui Merleau-Ponty parlava in termini di «geometria naturale», ovvero la possibilità che un oggetto inserito in uno spazio possa darmi il senso dell'orientamento di questo stesso spazio e in tal modo indirizzare il mio sguardo:

In generale la nostra percezione non comporterebbe né contorni, né figure, né sfondi, né oggetti, non sarebbe perciò percezione di nulla e infine non esisterebbe se il soggetto della percezione non fosse quello sguardo che ha presa sulle cose solo per un certo orientamento delle cose stesse [...]. (Merleau-Ponty 2003: 656-657)

Il compito del fotografo, secondo Ghirri, sta nel seguire l'orientamento di questi oggetti che sono già parte del paesaggio e giocare con le simmetrie ortogonali, con qualcosa che ci è dato, che abbiamo a disposizione. E proprio a questo gioco si riferisce Ghirri quando dice che le fotografie che ha fatto esistevano già nella realtà.

Negli anni della loro amicizia, anni in cui i percorsi individuali dei due autori inevitabilmente si intrecciarono anche su un piano professionale, Ghirri, secondo Marco Belpoliti, assume per Celati

la funzione di "figura guida", per dirla con un'espressione dello stesso scrittore, così come nel decennio precedente lo era stato Italo Calvino, suo mentore e sostenitore in Einaudi. La richiesta di Ghirri è di scrivere ciò che vede in giro per la pianura; in questo modo lo spinge a realizzare quelli che sono i "racconti d'osservazione". (Belpoliti 2016)

Quella «visione naturale», secondo Celati connaturata nella fotografia di Ghirri, caratterizzerà negli stessi anni anche la sua scrittura, specialmente le produzioni brevi - sarebbe forse meglio utilizzare il termine novelle - di Narratori delle pianure (Celati 1985). Nell'anno dell'uscita, Ghirri firma per il settimanale Panorama una recensione della raccolta $^{3}$, i cui racconti erano a suo dire caratterizzati da «un'attenzione non epidermica e frammentaria alle immagini del mondo esterno», a dimostrare come quello "sguardo stupito e rapito dall'esistente» costituisse un primo punto di distacco dai lavori precedenti. Scrive Elio Grazioli:

Ghirri mette a fuoco tutte le questioni comuni fondamentali. La prima emerge nell'evidenziare la differenza tra i libri precedenti di Celati e l'attuale: i primi «avevano privilegiato mondi personali e interiori, in questi racconti sono [invece]

3 La recensione di Luigi Ghirri, «Una carezza fatta al mondo», Panorama, giugno 1985, è oggi contenuta in Sironi 2004: 173-174. 
le voci ascoltate e gli spazi esplorati a essere protagonisti», cioè l'«esterno», il mondo. (Grazioli 2008: 148-149)

A quasi quindici anni di distanza dall'uscita della raccolta, nel luglio del 1999, Celati fu invitato a un convegno sulla novella, alla Fondazione Cini di Venezia. Durante l'incontro con Silvana Tamiozzo Goldman ${ }^{4}$, gli veniva chiesto di spiegare quanto la novellistica italiana tradizionale avesse a che fare con Narratori delle pianure, e in che modo avesse influenzato il suo lavoro. La novella tradizionale aveva solitamente una cornice d'apertura, la presenza di cerimonie introduttive che espongono la storia a partire dall'antefatto e che delineano anticipatamente il quadro della lettura che stiamo per intraprendere, contestualizzandone vicenda e personaggi. I racconti moderni, al contrario, cominciano quasi sempre in medias res, entrando subito nel vivo di un fatto a cui il lettore è estraneo, causando un senso di straniamento e la curiosità, al contempo, di ricostruire i fatti, di dare un volto ai nomi, di ambientarsi e districarsi nella complessità della storia. Con Narratori delle pianure, Celati si pone in controtendenza ${ }^{5}$ e prende a prestito un espediente tipico della novella tradizionale, rielaborandolo in chiave contemporanea. Egli recupera la cerimonia introduttiva per fornire l'argomento della novella, e ne fa la premessa temporale dei propri racconti.

«La novella si avvia sempre con questi piani panoramici, che ci avvicinano lentamente ai tempi puntuali del racconto» (Celati 2011a: 39), dice Celati. Il piano panoramico ha due enormi vantaggi: è volto al tempo imperfetto e non parte mai da una focalizzazione precisa, ma sempre da una vaghezza che permette di giocare con la trama, facendole prendere strade inattese, e che consente altresì di "far entrare in gioco voci improvvise d'altri tempi» (Celati 2011a: 40). Così la novella risulta come il racconto di un racconto già sentito e «celebra un sentito dire, che spunta da un passaggio di parole di bocca in bocca» (Celati 2011a: 40). Una struttura narrativa di questo tipo costringe a una considerazione sul narratore e sul livello a cui il suo racconto è collocabile:

Esistono casi in cui all'interno di un racconto principale viene infatti installato un racconto di «secondo grado» [...]. Tali forme di rappresentazione di una comunicazione narrativa all'interno del mondo diegetico [...] sono caratteristiche di quei testi che hanno una struttura detta «a cornice». (Bernardelli 1999: 41)

4 L'intervista cui si fa riferimento ha preso il titolo «Elogio della novella» ed è contenuta nella raccolta Conversazioni del vento volatore (Celati 2011a: 35-43).

5 La controtendenza celatiana non si limita al recupero degli elementi della novella tradizionale, ma si fa già tangibile nella scelta della forma breve come genere e mezzo della propria prosa. Durante la conversazione con Silvana Tamiozzo Goldman, Celati dirà che «I lettori, i critici vedono il racconto soltanto come qualcosa di più corto rispetto al romanzo, dunque più sbrigativo. Invece il racconto è un genere a sé, frammentario, dispersivo, nomade» (Celati 2011a: 35). Nella contemporaneità recuperare le forme della novella tradizionale è, in questo senso, sintomo di una necessità di narrare a prescindere dall'esito del racconto, senza l'urgenza di dare una giustificazione, una motivazione a ciò che si è scritto. 
Il narratore può quindi trasporre gli avvenimenti con maggiore o minore aderenza alla realtà, utilizzando "connotatori di mimesi o effetti di realtà» (Bernardelli 1999: 43), per far scaturire nel lettore la sensazione di essere davanti a un evento reale, effettivamente appreso da altri e riferito. Nei racconti celatiani è inoltre presente un gioco di testimonianze che, in alcuni casi, induce il pubblico a ipotizzare una sovrapposizione tra la fonte della storia e il narratore esterno.

Questo è un altro degli elementi che Celati reintroduce dalla novellistica cosiddetta tradizionale, e che rispetto ai suoi contemporanei egli sceglie di valorizzare, cioè il modo di rapportarsi e di comunicare col lettoreascoltatore, non basando la mutua fiducia sull'eventualità che la storia possa cogliere impreparati o soltanto stupire, creando un senso di sconcerto, ma al contrario ponendosi sullo stesso piano del pubblico, disvelando cautamente gli accadimenti:

Nelle novelle tradizionali la sorpresa non nasce da un segreto da svelare, né da un'informazione trattenuta, bensì da qualcosa di impensato che germoglia su un terreno in comune tra chi parla e chi ascolta. L'ascoltatore è messo al corrente dell'argomento e degli scopi del narrare con la cerimonia introduttiva, che non manca mai: "parliamo di questo". Ed è come quando si invita qualcuno nella propria casa, e la cerimonia dell'accoglimento colma le distanze dell'estraneità, portando l'altro su un terreno comune. (Celati 2011a: 38)

In Narratori delle pianure siamo accolti da una voce narrante che parte da una premessa fondamentale, ossia che il racconto è giunto fin lì da terzi e quello che ci apprestiamo a leggere è quindi più il ricordo, chissà quanto preciso, di una storia ascoltata altrove: «rispetto ai generi di maggior prestigio, come il poema epico, la lirica e l'oratoria, la novella si distingue per quest'altro tipo di circolazione, non chiuso negli spazi della pagina scritta» (Celati 2007: s.p.).

Celati apre il volume con una dedica che recita: «a quelli che mi hanno raccontato storie, molte delle quali sono qui trascritte» (Celati 1985:7) e in questa riga è già anticipato il senso che lega le trenta novelle. Entrando nel merito della raccolta, possiamo fare riferimento ad alcuni incipit che mi sembrano esplicativi di questa risonanza di voci di cui si è parlato e che è il mezzo di diffusione privilegiato delle narrazioni che nascono nell'oralità e che sono destinate a circolare come un sentito dire.

L'isola in mezzo all'Atlantico si apre con queste parole: «Ho sentito raccontare la storia di un radioamatore di Gallarate, provincia di Varese, il quale s'era messo in contatto con qualcuno che abitava su un'isola in mezzo all'Atlantico» (Celati 1985: 11). E ancora, Come fa il mondo ad andare avanti: «In un piccolo paese in provincia di Parma, non lontano dal Po, mi è stata raccontata la storia d'un vecchio tipografo che s'era ritirato dal lavoro perché voleva finalmente scrivere un memoriale a cui pensava da tanto tempo» (Celati 1985: 50). La presenza di una cornice, di un racconto nel racconto, provoca, anche in questo caso, la possibilità che i ruoli dell'autore e del lettore si invertano: chi scrive è stato, a suo tempo, il primo - o persino il secondo, o il terzo - ascoltatore di ciò che è intento a narrare e il lettore è, a sua volta, messo nella condizione di sentire per la 
prima volta una storia. Quella che Celati chiama «cerimonia dell'accoglimento» rappresenta un momento di indugio, di sospensione e apertura all'ascolto. Per fare un altro esempio, le righe di apertura de La macchina del moto perpetuo sono: "Un amico tedesco mi ha raccontato la storia di un operaio della Ruhr, che aveva progettato e cercava di costruire la macchina del moto perpetuo di seconda specie» (Celati 1985: 118). Quando non viene esplicitamente dichiarato se la storia sia giunta per bocca di una voce amica o prossima, il racconto assume i segni di una leggenda popolare o dell'eco di una storia che ha sempre aleggiato nelle campagne, o nei paesi di provincia. Penso anche a Cosa è successo a tre fratelli calciatori: "Questa è la storia di tre fratelli che si sono rivelati bravissimi giocatori di calcio in un torneo di squadre per ragazzi. Di loro si parlava in tutta la periferia dove abitavano.» (Celati 1985: 20) e a Sul valore delle apparenze: «C’è una donna non più giovane che svolge da moltissimi anni l'attività di domestica a ore in varie case borghesi di Cremona. Di lei si racconta che, appena uscita da un orfanotrofio, abbia incontrato un uomo chiamato "il calabrese"» (Celati 1985: 41).

Nelle Notizie sui testi disposte in chiusura al Meridiano Mondadori che raccoglie le prose celatiane, Nunzia Palmieri evidenzia che:

I primi abbozzi dei racconti che fanno parte della raccolta Narratori delle pianure si trovano in alcuni quaderni e agende di inizio anni Ottanta, dove Celati aveva iniziato a descrivere i luoghi che attraversava. [...] egli riempie i taccuini con appunti sul paesaggio, registrazioni di voci e atteggiamenti, ricordi, sogni, citazioni di testi letterari, note di lavoro, spunti narrativi. (Palmieri in Celati 2016: 1756)

Ancora una volta affiora l'idea di storie ascoltate, oppure addirittura osservate, colte nei gesti o nel parlare di persone sconosciute.

Nel 1984, Calvino aveva anticipato la raccolta in uscita scrivendo che Celati sarebbe tornato con un libro che "ha al suo centro la rappresentazione del mondo visibile, e più ancora una accettazione interiore del paesaggio quotidiano in ciò che meno sembrerebbe stimolare l'immaginazione» (Calvino 2008: 175). La presenza della doppia cornice, in questo senso, non tocca soltanto la diegesi della raccolta e il modello narrativo che Celati ha scelto di utilizzare per le trenta novelle - quindi il modo di orientare il lettore entro gli spazi narrativi - ma coinvolge anche gli spazi narrati. Come ha osservato Patrick Barron:

In effect, Celati here calls for a renewed attention to what Lefebvre terms "the truth of space" (concerning the spatial history of multidimensional everyday landscape) rather than "true space" (the two dimensional versions of ideal or designed space). (Barron 2007: 330)

Gli elementi spaziali assumono quindi una centralità, facendo da ulteriore filtro per il lettore, ovvero da soglia tra paesaggio e personaggio. Accade così che i protagonisti delle novelle possano avere familiarità coi luoghi della narrazione anche quando gli spazi rappresentati non sono quelli a cui effettivamente appartengono; pensiamo per esempio a L'isola in mezzo all'atlantico, e al momento in cui i due fidanzati raggiungono l'isola di Archie: 
Quando sono sbarcati hanno subito ritrovato la lunga strada che faceva un percorso circolare attorno al promontorio coperto d'erica. Riconoscevano quasi tutto e riuscivano a orientarsi come se ci fossero già stati. (Celati 1985: 13)

E alla stessa maniera il lettore è messo nella condizione di pensare di aver avuto esperienza diretta dei luoghi narrati, che, pur presentando elementi d'intangibilità - la nebbia che avvolge, sul finale, $i$ Bambini pendolari che si sono perduti, ma anche $\mathrm{i}$ "campi coltivati a grano, appezzamenti tutti uguali» descritti in Storia di un apprendistato - sono sempre descritti con dovizia di particolari. E questo avviene al punto che il racconto non paia più neanche soltanto una storia già sentita, ma un vissuto, spazi a cui il lettore è estraneo e che possono assumere i tratti della familiarità; prendiamo l'esempio del racconto Fantasmi a Borgoforte:

C'è una strada che a Borgoforte, provincia di Mantova, segue l'argine del Po fino ad un punto in cui il fiume Oglio si innesta nel Po, e lì sull'Oglio c'è uno dei rari ponti di barche rimasti in piedi, tra i tanti che esistevano in queste zone. Quella strada non è asfaltata, tranne per un tratto iniziale. Intorno ci sono molte vecchie case coloniche in rovina [...] e passando di lì dopo il tramonto è piuttosto difficile incontrare qualcuno, soprattutto nei mesi invernali quando quel viottolo sassoso lungo il fiume è avvolto da banchi di nebbia. In una sera di novembre due donne, impiegate nello stesso ufficio, tornavano a casa su quella strada e pioveva a dirotto. (Celati 1985: 63)

La dicotomia interno/esterno tocca quindi il lettore in prima persona: egli legge il racconto di un racconto già sentito e può a suo volta esserne testimone. Ma il concetto di doppio framing, come già accennato, può trovare facile applicazione anche nel cinema. Nel suo Il cinema e le arti visive, Antonio Costa ne parla in termini di "effetto quadro», prendendo a prestito l'espressione dal saggio $I$ pitto-film di François Jost, e porta l'esempio de I misteri del giardino di Compton House, «in cui l'effetto quadro è emblematizzato in un gioco di equivalenze tra il mirino della cinepresa e il riquadro ottico (la mira) usato dal pittore nel suo lavoro» (Costa 2002: 321). Fa inoltre ampio riferimento al saggio Décadrages di Pascal Bonitzer, che si è a lungo occupato di definire la relazione tra dispositivi visivi e spazio filmico, tanto da azzardare la definizione di «pittori» in riferimento ad alcuni noti registi:

È così dimostrata l'esistenza di analogie profonde tra organizzazione dello spazio e forme simboliche [...]. L'introduzione di inquadrature che introducono una «suspense non narrativa» è ciò che giustifica la definizione di «pittori» che Bonitzer attribuisce a cineasti come Antonioni, Straub e Huillet, Duras. (Costa 2002: 34)

Questi riferimenti possono essere utili a spiegare la presenza e la funzione del doppio framing nei lavori di Michelangelo Antonioni, regista che Costa definisce "pittore». Come noto, Celati è un estimatore di Antonioni, particolarmente del film L'avventura, intorno al quale si sviluppa il suo saggio, "La veduta frontale. Antonioni, L'avventura e l'attesa», oggi in Documentari imprevedibili come $i$ sogni (Celati 2011b: 29). L'avventura, film di Antonioni proiettato nelle sale nel 
1960 e, sempre nello stesso anno, in concorso al tredicesimo Festival di Cannes, costituisce il primo episodio della cosiddetta «Trilogia dell'incomunicabilità»e si apre con un gruppo di amici che, da Roma, parte per una gita in barca alle isole Eolie, nei pressi dell'isolotto roccioso e deserto di Lisca Bianca. In questa occasione Anna (Lea Massari) scompare nel nulla e il gruppo comincia a cercarla, specialmente Sandro (il suo compagno, interpretato da Gabriele Ferzetti) e la sua amica Claudia (Monica Vitti), vero perno di questa narrazione. La ricerca è lunga e vana, ma passa presto in secondo piano. I tempi, da serrati, si dilatano e l'attesa del ritorno di Anna diventa semplicemente il tempo dell'attesa che sovrasta l'inconsistenza della trama. I protagonisti vagano, inizialmente sull'isola, poi nelle città - prima Noto, poi Taormina - senza più essere realmente alla ricerca di qualcosa, soltanto preda di uno struggimento che da collettivo diviene personale e che nulla ha a che vedere con la scomparsa dell'amica. C'è molta insistenza, allora, sul profilo dell'isola, sugli eventi atmosferici che scuotono la storia e i suoi protagonisti:

In questo film il paesaggio è una componente non solo indispensabile, ma quasi preminente. Ho sentito il bisogno di spezzettare molto l'azione, inserendo in molte sequenze inquadrature che possono sembrare formalistiche o non essenziali, inquadrature di tipo addirittura documentario (una tromba d'aria, il mare, il passaggio dei delfini, cose di questo genere), ma che in realtà per me sono indispensabili, perché servono l'idea del film. (Antonioni 2009: 17)

Antonioni sosteneva che fosse necessario avvicinarsi al personaggio partendo dalle cose piuttosto che dalla sua vita, secondo un procedimento che va «dal particolare all'insieme»:

Partendo da particolari che mi colpiscono risalgo a situazioni d'insieme. Quando mi piace qualcosa di un luogo, mi viene subito l'idea di muovervi dei personaggi. (Antonioni 2009: 218)

Nel suo saggio, Celati ripensa al film a partire dall'uso che Antonioni fa della ripresa frontale, che «consente indugi senz'ansia, che spesso sono proprio tempi morti sul filo della narrazione» e che "è essenzialmente la scelta di una bassa soglia di intensità, d'un modo di narrare che evita le eccitazioni, e riporta tutto ad un pacato uso della rappresentazione» (Celati 2011b: 29). Altro elemento caratterizzante del film è, però, l'insistenza sulle inquadrature di quinta, cioè le riprese effettuate da sopra la spalla di un soggetto, con la schiena utilizzata per incorniciare l'immagine. Osserva Tinazzi che «nella descrizione dilatata degli ambienti, la macchina sembra tallonare i personaggi, quasi a smascherarne l'ambivalenza» (Tinazzi 1974: 49). L'avventura presenta molte inquadrature di questo genere, quasi sempre accompagnate dalla presenza di una finestra, di una porta, oppure di un balcone. Separano gli interni delle case e dei palazzi dagli esterni, oppure fungono da soglia per i protagonisti che immobili osservano scene a cui non prendono parte. Servono a creare attesa e tempi morti in cui i soggetti si limitano a guardare, o a riflettere, senza che nulla accada. Si pensi alla scena in cui Claudia (Monica Vitti) si affaccia dalla finestra, mentre sullo sfondo l'amica Giulia e il giovane pittore Goffredo dialogano, cercando di 
coinvolgerla in un discorso a cui prontamente si sottrae. Niente riesce a distrarla, tanto è presa da quell'osservazione insistente sull'affaccio verso l'esterno, sulla vegetazione e sui suoni che insistono dall'altro capo della finestra, e neppure lo spettatore può riuscire a provare interesse, d'un tratto, per le parole di Giulia e Goffredo, focalizzando inevitabilmente tutta l'attenzione su Claudia, sul suo corpo voltato e teso verso il vuoto, nel tentativo di comprendere, di ipotizzare cosa stia pensando. Parlando della sua esperienza di regista e della tecnica di ripresa, Antonioni afferma:

Credo che l'inquadratura sia una questione figurativa, che deve essere vista nella sua giusta dimensione. La recitazione ha valore in rapporto all'inquadratura: una battuta detta da un attore di spalle è diversa dalla stessa battuta detta di faccia o di profilo: assume un altro valore, un altro significato. (Antonioni 2009: 9)

Queste scene servono dunque anche allo spettatore per sentirsi dentro il film e direttamente affacciato sulla scena, per avere la percezione dell'ineluttabilità del tempo o al contrario per provare un senso di distacco dagli accadimenti, per rimanere al di fuori della cornice e dei fatti. Le cornici che separano gli interni dagli esterni rappresentano l'albertiana «fenêtre ouverte sur le monde» a cui faceva riferimento André Bazin, e di cui Jacques Aumont parla ne L'œil interminable:

Perspective ou pas, la fenêtre ouverte d'Alberti et de ses héritiers ouvre sur un monde lisible, et le tableau n'en finit pas de délivrer des signifiés. (Aumont 2007: 127)

L'ambiente era sì, per Antonioni, un contorno, ma era anche l'unica possibilità di mettere le cose, «la materia», in contatto coi personaggi, così che avessero sempre «un occhio aperto sull'esterno e uno ripiegato all'interno» (Antonioni 2009: XXXIII). Escludere o includere nell'immagine un particolare anche apparentemente secondario era una «decisione essenziale per la riuscita di un film» (Antonioni 2009: 131).

Anche Giorgio Tinazzi individua nella poetica di Antonioni la forte presenza di una dicotomia interno-esterno, un limite nuovamente inteso come ago della bilancia tra una spinta del regista "verso l'introiezione (le psicologie, ciò che con facile definizione si disse il suo neorealismo interiore)» (Tinazzi 1974: 34) e quella, antitetica, che lo conduce verso il fuori e che restituisce importanza all'ambiente, ai mutamenti del paesaggio, agli oggetti e ai tempi morti. E in Antonioni, scrive Celati, "sono appunto i tempi morti, gli sguardi o i gesti d'indugio senza meta a riaprire per noi questa comprensione» (Celati 2011b: 29), una comprensione in cui tutto diventa osservabile, in cui tutti i luoghi sono degni di un'attenzione, in cui tutto può essere soglia della nostra riflessione. Secondo Costa è inoltre possibile collegare particolari paesaggi a una particolare «stimmung»:

diventano spazi intercambiabili: in essi, si configura una modalità di sguardo che è insieme di estraneità e inquieta partecipazione, di esclusione e di inclusione. Le immagini di paesaggio nel cinema di Antonioni sono sempre immagini sospese: immagini dotate di una suspense non narrativa [...] Al pari del primo piano, 
anche gli «spazi qualunque» rientrano nella tipologia dell'immagine affezione.

(Costa 2002: 347-348)

Gli «spazi qualunque», di zavattiniana memoria, sono il luogo privilegiato di questo «moto pendolare tra narrare e mostrare» che accomuna il lavoro di Antonioni, Celati e Ghirri, e il doppio framing altro non è che un espediente visivo, architettonico o strutturale, di cui servirsi per operare lo scarto tra la parvenza e la rappresentazione dello spazio e dei personaggi, lo strumento che permette all'autore di «ritagliare una finestra mentale» (Ghirri 2010: 156) e dare al proprio pubblico un elemento di accesso alla visione del mondo esterno. E quello che tutti e tre sono stati in grado di fare, ognuno nel proprio terreno di gioco, è ciò che Ghirri ha definito un lavoro profondo sul sistema di rappresentazione, «sulla scoperta di una realtà che è già presente all'interno della realtà».

\section{Bibliografia}

Antonioni M., 2009, Fare un film è per me vivere, Venezia, Marsilio Editori.

Aumont J., 2007, L'œil interminable, Parigi, Éditions de la Différence.

Bachelard G., 1957, La poetica dello spazio, Bari, Dedalo.

Barron P., 2007, «Gianni Celati's Poetic Prose: Physical, Marginal, Spatial», Italica, Vol. 84, n. 2/3, p. 323-344

Bazzocchi M., 2012, L'Italia vista dalla luna. Un paese in divenire tra letteratura e cinema, Milano, Bruno Mondadori.

Bernardelli A., 1999, La narrazione, Bari, Editori Laterza.

Calvino I., [1985] 2008, «Da Buster Keaton a Peter Handke», Riga, 28, Gianni Celati, a cura di M. Belpoliti e M. Sironi, p. 175.

Celati G., 1985, Narratori delle pianure, Milano, Feltrinelli.

Celati G., 2007, «Lo spirito della novella», Griseldaonline, n. VI.

Celati G., 2011a, Conversazioni del vento volatore, Macerata, Quodlibet.

Celati G., 2011b, Documentari imprevedibili come i sogni. Il cinema di Gianni Celati, a cura di N. Palmieri, Roma, Fandango.

Celati G., 2016, Romanzi, cronache e racconti, a cura di M. Belpoliti e N. Palmieri, Milano, Mondadori.

Costa A., 2002, Il cinema e le arti visive, Torino, Einaudi.

Ghirri L., 2010, Lezioni di fotografia, Macerata, Quodlibet.

Merleau-Ponty M., 2003, Fenomenologia della percezione, Milano, Bompiani.

Grazioli E., 2008, «Gianni Celati e Luigi Ghirri», Riga, 28, Gianni Celati, p. 143-154.

Norberg-Schulz C., 1992, Genius Loci. Paesaggio, ambiente, architettura, Milano, Electa.

Simmel G., 1997, «La cornice del quadro. Un saggio estetico», in I percorsi delle forme. I testi e le teorie, a cura di M. Mazzocut-Mis, Milano, Bruno Mondadori. 
Sironi M., 2004, Geografie del narrare. Insistenze sui luoghi di Luigi Ghirri e Gianni Celati, Reggio Emilia, Diabasis.

Somaini A., 2000, «La cornice e il problema dei margini della rappresentazione», Le parole della filosofia, n. III.

Stoichita V.I., 1998, L'invenzione del quadro, Milano, Il Saggiatore.

Tinazzi G., 1974, Michelangelo Antonioni, Milano, Il Castoro. 